\title{
Conciencia metapragmática y memoria operativa en niños escolares*
}

\author{
Nina Crespo Allende** \\ Carola Alvarado Barra***
}

\section{Resumen}

La conciencia metapragmática se define como la capacidad que posee un sujeto para establecer relaciones entre un mensaje lingüístico oral y su contexto extralingüístico. Gombert (1992) sostiene que su desarrollo está influenciado por la memoria de trabajo. En este marco, la presente investigación busca determinar si existe una relación estadísticamente significativa entre la conciencia metapragmática y la memoria operativa. Para ello, se midieron ambas variables en 88 niños y niñas entre los 8 y 9 años de edad. Los resultados indican una correlación positiva, lineal y significativa y, de esta manera, se comprobó que el incremento de la conciencia metapragmática no depende solamente del desarrollo lingüístico de los individuos, sino también de una maduración de los sistemas cognitivos involucrados en el almacenamiento y procesamiento de la información.

Palabras Clave: Metalingüística, conciencia metapragmática, memoria operativa

\begin{abstract}
Metapragmatic awareness is defined as the ability to establish relations between an oral linguistic message and its extralinguistic context. Gombert (1992) argues that its development is influenced by the working memory. In line with the above, this investigation seeks to determine whether or not there is a statistically significant relation between metrapragmatic awareness and working memory. To that purpose, both variables were measured in 88, 8-9-year-old boys and girls. Findings indicate a positive, linear, and significant correlation, thus proving that an increase in metapragmatic awareness depends not only on people's linguistic development, but also on the maturity of the cognitive systems involved in the storing and processing of information.
\end{abstract}

Key Words: Metalinguistics, metapragmatic awareness, working memory

* Investigación realizada en el marco del proyecto FONDECYT 1070333

** Doctora en Lingüística. Académica Pontificia Universidad Católica de Valparaíso. ncrespo@ucv.cl

*** Magíster en Lingüística Aplicada, Pontificia Universidad Católica de Valparaíso. carola_alvarado@mi.cl 


\section{Introducción}

La conciencia metapragmática se define como la capacidad que posee un sujeto para establecer relaciones entre un mensaje lingüístico oral y su contexto de uso (Gombert, 1992), es decir, implica el ajuste del emisor o destinatario del enunciado con los parámetros contextuales en el cual este se produce. Dicha habilidad le permitiría al hablante lidiar con usos cada vez más sofisticados del lenguaje y estaría basada en un conocimiento cada vez más creciente respecto del sistema lingüístico y de las convenciones de su uso (Verschueren, 2000; Levorato \& Cacciari, 1992).

Pero, ¿cómo se desarrolla esta habilidad?, ¿qué elementos inciden en su emergencia y posterior evolución? La problemática de esta investigación surge a partir de lo propuesto por Gombert (1992) acerca de qué elementos intervienen en el desarrollo de esta capacidad. Para este autor, si bien la conciencia metapragmática aumenta de acuerdo a la edad, ésta también tendría una directa relación con el aumento en la capacidad de procesamiento de la memoria de trabajo (Crespo, 2008). Bajo este precepto, el autor francés considera que la conciencia metapragmática, a pesar de ser una habilidad propiamente lingüística, dependería también de dicha característica estructural del sistema cognitivo. Lo anterior se explicaría porque esta capacidad metalingüística, aún en sujetos de mayor edad, no opera siempre de manera sistemática, sino que su fiabilidad dependería de la complejidad del input. Es decir, un sujeto de cierta edad (más de 7 u 8 años) que se da cuenta de la ambigüedad de un enunciado respecto a su contexto, se confunde si el input se vuelve más complejo (por ejemplo, aumentando el número de referentes), ya que ello superaría la capacidad de su memoria operativa.

Ahora bien, las pruebas que presenta Gombert (1992) para sostener tal afirmación solo se remiten a estudios aislados de laboratorio y carecen de una fundamentación empírica mayor. Así pues, dentro de este marco contextual, el presente estudio tiene como objetivo determinar si existe una relación estadísticamente significativa entre conciencia metapragmática y memoria de trabajo u operativa. Para ello, dentro de los objetivos específicos de esta investigación contempla medir, por un lado, la conciencia metapragmática, tal como Gombert (1992), Verschueren (2000; 2002) y Crespo (2008) la definen y, por otro, la capacidad de memoria operativa, asumiendo como fundamento teórico el modelo working memory de Baddeley y Hitch (1974).

\section{Marco Teórico}

\section{Conciencia Metapragmática}

La conciencia metapragmática es definida por Gombert (1992) como la conciencia y conocimiento que un sujeto posee acerca de las relaciones que existen dentro del sistema lingüístico, así como de las que se dan entre este sistema 
y el contexto en el cual el lenguaje se inserta. En este marco conceptual, lo metapragmático incluiría el control intencional de los parámetros lingüísticos que determinan la eficiencia del mensaje, en concordancia con aspectos extralingüísticos de la situación en la cual el enunciado se presenta.

Al respecto, Verschueren $(2000,2002)$ argumenta en sus trabajos de que todo uso del lenguaje implica un nivel de conciencia metapragmática. Es decir, cada vez que un sujeto construye un mensaje o lo interpreta, realiza una serie de elecciones lingüísticas, tomando en consideración el rol mediador de las mentes tanto del enunciador como del intérprete. En este sentido, los hablantes saben más o menos lo que hacen cuando están usando el lenguaje. Este conocimiento supone para el usuario estar al tanto de que posee una gama de posibilidades lingüísticas para elegir y esta elección la ejecuta de manera consciente. Por ello, el autor belga afirma que todas las elecciones lingüísticas, que realiza un sujeto cuando modifica un enunciado, tienen algún grado de conciencia.

De ahí que es importante destacar que, bajo esta perspectiva y tomando como punto de partida al emisor, la conciencia metapragmática es comprendida como aquella capacidad que posee el sujeto para elegir ciertas posibilidades lingüísticas que permita a su interlocutor llegar a un estado mental acorde con la intención que se quiere comunicar (Portolés, 2004). Se trata, por tanto, de la conciencia de cómo se usa el lenguaje, relacionado con la capacidad de elección de una formulación específica en cada momento de la comunicación. En este sentido, Portolés (2004) señala que los hablantes de una lengua tienen ciertos conocimientos pragmáticos más o menos conscientes que les permiten elegir formulaciones lingüísticas consideradas como más oportunas en situaciones comunicativas específicas. Por consiguiente, cada elección representa una reflexión sobre el propio lenguaje (Portolés, 2004; Verschueren, 2002).

Ahora bien, volviendo a la definición de Gombert (1992) señalada antes, los estudios sobre la conciencia metapragmática se han enfocado en determinar la relación entre los signos del sistema lingüístico y el contexto del enunciado, es decir, aquel que abarca tanto lo intra como extralingüístico. Respecto a cómo se desarrolla esta habilidad, este mismo autor ha determinado una incidencia importante de la edad en las habilidades de ajuste entre el mensaje lingüístico y los parámetros contextuales de la situación comunicativa. En este sentido, Gombert (1992) sostiene que esta conciencia metapragmática se iría logrando de manera más adecuada a medida que aumenta la edad de los sujetos. Ante tal incidencia, Gombert (1992) plantea que existirían dos fases (no etapas) recursivas que se presentarían en el desarrollo de la conciencia metapragmática. Estas serían: la fase epipragmática y la metapragmática.

Por una parte, la fase epipragmática corresponde a aquella donde tienen lugar aquellas conductas que surgen en edades muy tempranas (2- 3 años de edad) y en las cuales el niño adapta las producciones lingüísticas al contexto donde fueron emitidas. Gombert (1992) sostiene que dichas habilidades permiten probar la incapacidad del menor de disociar el lenguaje de su con- 
texto de emisión, sin embargo, no representan una identificación conciente de las relaciones que existen entre lo lingüístico y lo extralingüístico, porque en ningún momento hay una distinción entre ambas. Bajo esta definición, se puede establecer que los comportamientos epipragmáticos se caracterizan por su carácter incidental de la adaptación del lenguaje a su contexto, es decir, constituyen un uso sin una intención de control conciente del lenguaje, sino de un ajuste automático.

Por otra parte, la fase metapragmática corresponde a aquella donde se manifiesta una reflexión o control conciente del lenguaje en relación al contexto de emisión. Es decir, las acciones del sujeto permiten reconocer una capacidad de monitoreo de lo dicho en función al contexto de enunciación. Dicha capacidad se presentaría alrededor de los 6 y 7 años edad, desarrollándose hasta la adolescencia. Al respecto, Gombert (1992) plantea que la posibilidad de considerar organizaciones cada vez más complejas de referencias contextuales y de responder por medios de organizaciones lingüísticas cada vez más sofisticada depende, en gran medida, por el crecimiento o desarrollo de la capacidad de procesamiento de la memoria de trabajo.

\section{Memoria operativa o de trabajo}

La memoria operativa o de trabajo (working memory) es definida por Baddeley y Hitch (1974) como aquel sistema tripartito de almacenamiento y procesamiento de información temporal. Ante tal definición, dichos autores plantean en su modelo una memoria formada por tres componentes. En primer lugar, el sistema ejecutivo central de capacidad limitada y cuya función es supervisar y coordinar los otros subsistemas. En segundo lugar, el bucle o lazo fonológico, subsistema especializado en el almacenamiento temporal de la información basada en el lenguaje. En último lugar, la agenda viso-espacial, encargada del almacenamiento temporal de la información visual y espacial (Ver Fig.1)

Figura 1. Modelo de memoria de trabajo (Baddeley \& Hitch, 1974)

\section{AGENDA VISO-ESPACIAL EJECUTIVO CENTRAL}

\section{BUCLE FONOLÓGICO}

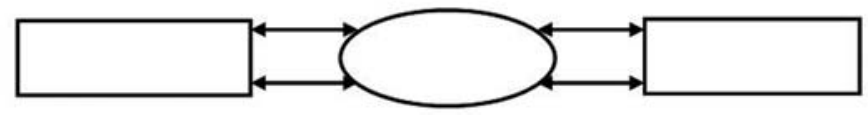

\section{Ejecutivo central}

Sistema que controla y supervisa dos subsistemas: el bucle fonológico y la agenda visoespacial. Por ello, su funcionabilidad apunta más a la atención que al almacenamiento de información (Baddeley, 1999). Por su parte, Gathercole y Pickering (2000) sugieren otras funciones adscritas al ejecutivo central. Estas 
apuntan al desarrollo de estrategias flexibles para el almacenaje y la recuperación de la información; al control del flujo de información a través de la memoria de trabajo, a la recuperación del conocimiento desde la memoria a largo plazo, o al control de la acción, la planificación y la programación de múltiples actividades concurrentes (Alsina, 2007).

\section{Bucle fonológico}

El bucle fonológico es el componente que más se ha desarrollado en el modelo de Baddeley y Hitch de 1974 (Alsina, 2007). Este subsistema está constituido por dos componentes: (a) Un almacén fonológico, con capacidad para retener información basada en el lenguaje; (b) Un proceso de control articulatorio, basado en el habla interna. Sobre este sistema, Baddeley (1999) plantea que las huellas de memoria en el almacén fonológico se desvanecen y resultan irrecuperables después de un segundo y medio o dos, aproximadamente. Sin embargo, esta huella puede reactivarse por un proceso de lectura de la huella dentro del proceso de control articulatorio, el cual vuelve a alimentar entonces el almacén. Esto explicaría la eficacia de la estrategia de repetición para favorecer el almacenamiento (Alsina, 2007). Asimismo, este componente permite ofrecer una explicación coherente a diferentes fenómenos empíricos (Baddeley, 1999): el efecto de similitud fonológica, el efecto del habla no atendida, el efecto de longitud de palabras y la supresión articulatoria.

\section{Agenda viso-espacial}

En el modelo de 1974, el componente viso-espacial se consideraba un sistema similar al bucle fonológico, pero dedicado a la información de carácter visual, aunque en aquel momento todavía no había suficiente evidencia empírica (Baqués, 1996). Actualmente, el segundo gran sistema subordinado postulado por el modelo de memoria de trabajo es aquel que se encarga de crear y manipular imágenes viso-espaciales y el cual se emplea en la creación y utilización mnemotécnicas de imágenes visuales (Baddeley, 1999). A la vez, los investigadores han determinado que al parecer el sistema espacial es importante para la orientación geográfica y para la planificación de tareas espaciales, aunque no parece encargarse del efecto de la imaginabilidad en la memoria verbal a largo plazo (Baddeley, 1999). Gracias a diversas evidencias provistas de investigaciones neuropsicológicas, se ha podido establecer que la agenda viso-espacial se compone de dos subsistemas independientes. Uno encargado del procesamiento de patrones y de detectar el qué; y, otro, relativo a la localización en el espacio que transmite información sobre el dónde (Baddeley, 1999; Gathercole \& Pickering, 2000; Alsina, 2007).

El modelo inicialmente propuesto por Baddeley y Hitch (1974) y sus reformulaciones posteriores, ha generado y sigue generando distintos trabajos que demuestran que a nivel conceptual todavía no puede ser considerado un modelo cerrado. Este argumento se apoya en el hecho que en el transcurso de 
los últimos años han surgido algunas propuestas alternativas, entre ellas, las de Case (1985), Engle, Cantor y Carullo (1992) y Salthouse (1990).

\section{La memoria de trabajo y su rol en la conciencia metapragmática}

Para referirnos a la influencia de la memoria operativa en la comprensión oral, retomaremos el concepto de metapragmática planteado en líneas anteriores y considerado por autores como Verschueren (2000) como la habilidad que permite al emisor y al destinatario un uso adecuado de esta modalidad. En este sentido, Gombert (1992) plantea que uno de los elementos claves que determinarían la conciencia metapragmática sería la memoria de trabajo. Ello se justifica, ya que, si bien los niños menores no parecen identificar el carácter potencialmente ambiguo de los mensajes, el desempeño de los niños mayores parece depender de la complejidad del mismo. Así, por ejemplo, en una tarea de identificar referentes ambiguos, mientras mayor cantidad de referentes hay implicada en ella, más dificultad tendría el menor en identificar la ambigüedad de la información (Crespo, 2008).

Frente a estos resultados, se establece que puesto que la única diferencia entre los ítems de la tarea es el volumen de la información que tiene que ser manejada en la memoria de trabajo, la explicación en términos de capacidad de procesamiento es la que mejor explica el mejoramiento del desempeño como una función de la edad (Gombert, 1992). En otras palabras, los niños mayores procesan mejor que los menores, porque su capacidad de procesamiento ha evolucionado y -además- esta capacidad se ve afectada por la complejidad del input.

De igual forma, algunos autores postulan que esta consideración también puede ser atribuida a tareas que requieren la formulación de solicitud de información adicional para mayores clarificaciones, como son aquellas en las cuales se dan instrucciones ambiguas o incompletas. Al respecto, Brown (1987) demostró que si bien los adolescentes de 14 años de edad son capaces de detectar la ambigüedad del lenguaje, tienen gran dificultad para formular solicitudes adecuadas de información adicional. Los autores asocian dicha dificultad a problemas en la capacidad de memoria de trabajo.

Asimismo, otro aspecto de la conciencia metapragmática se evidencia en la producción oral e implica adaptar el habla al interlocutor, habilidad considerada fundamental en el desarrollo del lenguaje oral tardío (Ravid \& Tolchinsky, 2002). Acuña y Sentis (2004) diferencian dos tipos de adaptación. Una de ellas es la comunicación de referencias, es decir, cuando el niño formula su discurso se adecua a las necesidades del oyente (lo que sabe, lo que piensa, etc.). Esta habilidad se desarrolla lentamente y es muy incipiente entre los 3 y los 6 años. Luego, se enriquece considerablemente entre los 7 y los 12 años. La otra es llamada por los autores cambio de código, e implica cambiar el estilo 
dependiendo del interlocutor. Respecto de ella, los autores la señalan como más temprana, ya que, pequeños de tres años son capaces de hacer adaptaciones cuando hablan con niños menores.

¿Cómo se relacionarían estas adaptaciones con la conciencia metapragmática? Gombert (1992) había señalado un distingo: mientras la primera obliga a un ejercicio más consciente e involucra un control metapragmático de la producción, la segunda sería más automática y estaría realizándose a un nivel epipragmático. De esta manera, mientras la primera estrategia sí exigiría mayor capacidad de memoria operativa y, por lo tanto, se desarrollaría en forma más tardía cuando aumenta la edad del sujeto; la segunda tendría un carácter más automático y no implicaría necesariamente una gran capacidad de memoria de trabajo. En este sentido, lo que se plantea en las anteriores afirmaciones es que existe una relación significativa entre el desarrollo de la conciencia metapragmática y el sistema de memoria operativa los que pretendemos corroborar en este estudio.

\section{Metodología}

Este estudio se enmarca dentro de un enfoque cuantitativo de alcance correlacional, pues su objetivo es evaluar la relación que existe entre dos variables: la memoria de trabajo y la conciencia metapragmática. Para llevar a cabo este objetivo, se utilizó un diseño no experimental transeccional. En consideración a lo señalado en el marco teórico acerca del momento clave del desarrollo de la memoria de trabajo y de la emergencia de la conciencia metapragmática, se trabajó con 88 niños y niñas de Tercer Año de Educación Básica, cuyas edades fluctuaban entre los 8 y 9 años de edad, de tres colegios de la comuna de Viña del Mar, dos particulares y uno subvencionado.

Para efectos de esta investigación, se utilizaron tres instrumentos de medición. Por una parte, dos test para medir memoria operativa. Por otra, un software llamado CMP, elaborado en el marco del proyecto FONDECYT 1070333, que mide conciencia metapragmática. Las pruebas que midieron MO fueron extraídos de la Batería de Memoria de Trabajo de Pickering, Baqués y Gathercole (1999).

Por una parte, la prueba Recuerdo Serial de Dígitos (Inverso) es considerada como un test que mide la habilidad del ejecutivo central, debido a sus requerimientos de mantener una lista de dígitos a través del recuerdo serial e invertirlo mentalmente. Esto indica que se realiza a la vez almacenamiento (el sujeto debe recordar los dígitos) y procesamiento (el sujeto debe invertir la serie de dígitos). Este test dispone de cuatro secuencias de dígitos para cada nivel y se suspende su administración cuando el sujeto falla en dos series de una misma amplitud. La puntuación se obtiene contando el número de ensayos correctamente recordados hasta la finalización del test. 
Por otra parte, la prueba Amplitud de Escuchar, así como en la prueba anterior, su tarea involucra de forma simultánea el mantenimiento y el procesamiento de la información. Se trata de una versión del Reading Span Task (test de amplitud de lectura) de Daneman y Carpenter $(1980,1983)$ en su modalidad de escuchar en lugar de leer (Listening Span Task). Consiste en la lectura de unas series de frases por parte del evaluador que el sujeto debe indicar si son verdaderas (V) o falsas (F). Una vez que se le ha leído las series y el sujeto ha respondido $\mathrm{V}$ o F, se le pide que repita la última palabra de cada frase, mediante recuerdo serial. La tarea se inicia con series de dos frases, por lo que una vez finalizada la serie, el sujeto debe recordar dos palabras. Se prosigue con una serie de tres frases y así, sucesivamente, hasta que el niño es incapaz de recordar correctamente y en el mismo orden, las últimas palabras de dos series de una misma amplitud. Debido a que el procedimiento es bastante complejo, es necesario realizar previamente al inicio del test un buen entrenamiento de la tarea. La puntuación final se obtiene contando el número total de series en las cuales el sujeto ha sido capaz de recordar correctamente todas las palabras finales en el orden correcto.

Respecto al instrumento dirigido a medir conciencia metapragmática, el CMP, corresponde a un software multimedial, creado por Crespo, Pérez y Alfaro (2008) en el marco del Proyecto FONDECYT 1070333. Dicho instrumento consta de tres ejercicios distribuidos en 29 ítems, y en los cuales se les pide a los sujetos dar cuenta acerca de desajustes evidentes entre el mensaje lingüístico y el contexto donde fue enunciado (Crespo, 2008). Estos son: Referente Ambiguo, Instrucciones Ambiguas y Diálogo Observado. De acuerdo a las pruebas estadísticas, el CMP es un instrumento altamente confiable (0,88 en el Coeficiente del Alpha de Cronbach) para medir conciencia metapragmática en niños de ocho años de edad (Montenegro \& Pérez, 2007; Crespo, 2008).

\section{Análisis de los Datos}

\section{Medición de la conciencia metapragmática: Análisis descriptivo}

La media o promedio de los sujetos evaluados con el CMP (23) corresponde al 39\% de la puntuación máxima de esta prueba (58) como se muestra en el gráfico 1. El rango entre el puntaje mayor (48) y el puntaje menor (8) obtenido por los sujetos en el CMP es de 40 puntos. La desviación estándar corresponde al promedio de desviación de las puntuaciones con respecto a la media. En este caso, la desviación estándar de las puntuaciones generales respecto a la media es de 10,5. 
Gráfico 1. Porcentaje del Promedio General del CMP

Porcentaje Promedio Rendimiento CMP

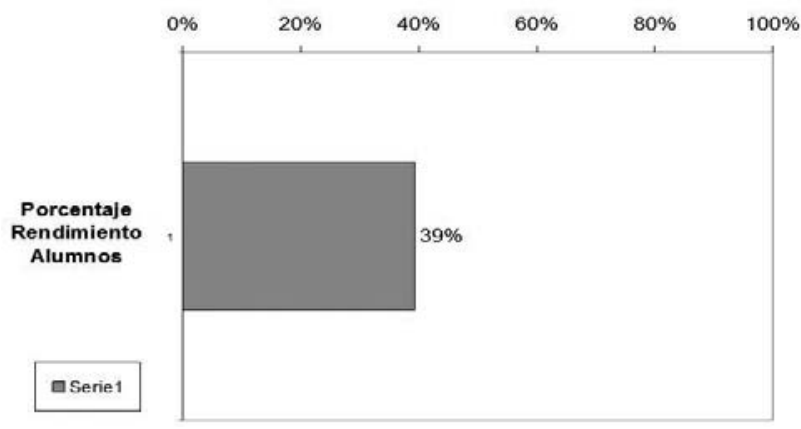

Desde el punto de vista de la distribución de los puntajes obtenidos, se establece que el 50\% de los sujetos evaluados por el CMP tuvo puntaje igual o superior a 20 puntos, es decir, 3 puntos menos que el puntaje medio. De esta manera, se determina que la muestra presenta una asimetría positiva en torno al valor medio estimado, lo que significa que hay más valores por debajo de la media. En otras palabras, el CMP constituyó una prueba de alta dificultad para niños y niñas de 8 y 9 años de edad. Respecto del puntaje total de la prueba, parcializado según el tipo de ejercicio, en la Tabla 1 se presentan los promedios obtenidos por los sujetos en cada ítem, así como el puntaje mínimo y máximo obtenido en cada uno de ellos.

Tabla 1 y Gráfico 2. Síntesis de puntajes promedio por tipo de ejercicio del CMP

\begin{tabular}{|l|c|}
\hline TIPO DE PREGUNTA & MEDIA \\
\hline Referente Ambiguo & 5 \\
\hline Instrucciones Ambiguas & 6 \\
\hline Diálogo Observado & 12 \\
\hline TOTAL & 23 \\
\hline
\end{tabular}

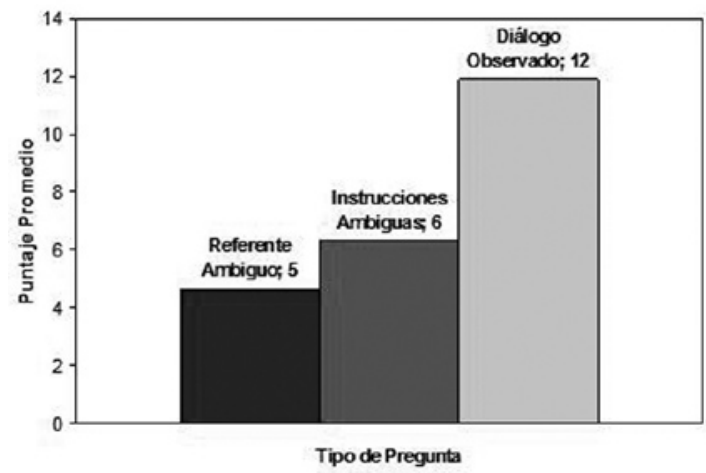


Considerando los puntajes totales por tipo de pregunta, se aprecia en la Tabla 8 que las preguntas de tipo Referente Ambiguo presentan un puntaje medio de 5 puntos, con el $50 \%$ de los alumnos con puntuaciones iguales o superiores a 2 puntos, y su variabilidad respecto a la media es de $s=5,2$. Esto indicaría una mayor dificultad con respecto a los otros dos tipos de preguntas entre los alumnos evaluados. Los ejercicios de Instrucciones Ambiguas presentan un puntaje medio de 6 puntos, con el $50 \%$ de los alumnos con puntaje igual o superior a 5 y con una variabilidad respecto al puntaje promedio de $s=5,3$. Finalmente, las preguntas de menor dificultad estarían constituidas por las preguntas de Diálogo Observado con un promedio de 12 puntos, donde el $50 \%$ de los alumnos presentan un puntaje igual o superior a 11 puntos, y su variabilidad corresponde a $s=4,5$.

Ahora bien, este análisis de puntajes totales debe ser profundizado con un enfoque que de cuenta del tipo de respuesta que dan los niños. Como se ha señalado en la descripción del instrumento, el CMP mide la conciencia metapragmática atendiendo a fases recursivas en las que el sujeto puede estar transitando al mismo tiempo (Crespo, 2008). Se puede plantear que, tanto las respuestas de puntuación 0 y 1, constituyen un comportamiento epipragmático, y que las repuestas de puntuación 2 a lo metapragmático. En el Gráfico 3 se presenta el porcentaje de estas respuestas.

Gráfico 3. Porcentajes de Respuestas Epipragmáticas y Metapragmáticas

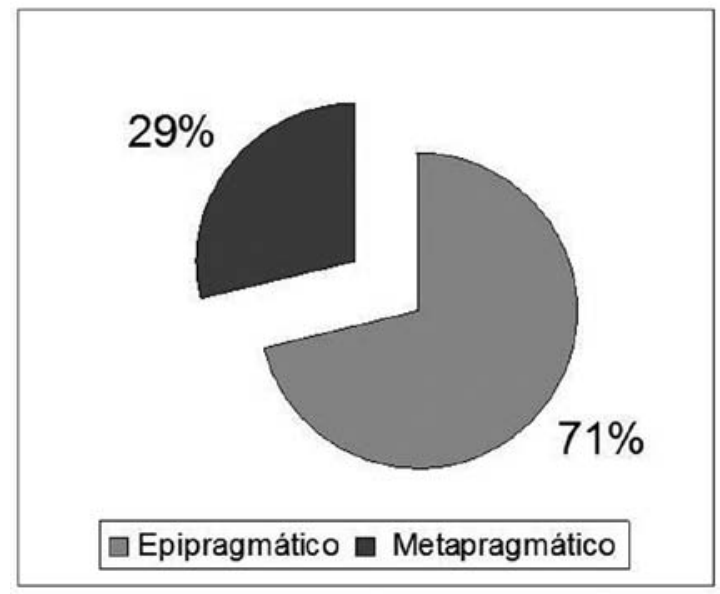

Frente al Gráfico 4, se puede determinar que en la mayoría de las respuestas, los niños todavía evidencian un nivel epipragmático de comprensión, ya que prevalecen las respuestas de 0 y 1 puntos. No obstante, dado que cada tipo de ejercicio obligaba al sujeto a establecer relaciones diferentes entre el contexto y el mensaje lingüístico se consideró observar qué ocurría con estas repuestas al interior de cada ítem. Esta información se presenta en el Gráfico 4. 
Gráfico 4. Porcentaje de Respuesta Epipragmáticas y Metapragmáticas por tipo de ejercicio

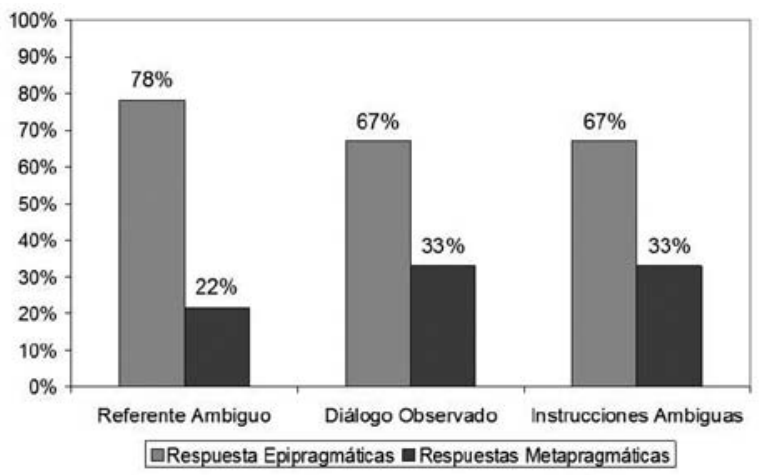

Si se analiza la distribución de puntajes presentados en el Gráfico 4, se observa en todos ellos que prevalecen las respuestas epipragmáticas por sobre las metapragmáticas. Sin embargo en Referente Ambiguo, el ítem más difícil si se consideran los puntajes totales, esta diferencia a favor de lo epipragmático (57\%) es mayor que en los otros ejercicios (34\%, 35\%).

\section{Medición de la memoria operativa: análisis descriptivo}

Con el fin de describir los datos recogidos en las pruebas que miden memoria operativa, se presentan a continuación las medidas de tendencia central (valores medios o centrales) y de variabilidad de estos test. En la Tabla 2, se reúnen los datos de la prueba de Recuerdo Serial de Dígitos (inverso).

Tabla 2. Medidas de tendencia central y variabilidad del test Recuerdo Serial de Dígitos (Inverso)

\begin{tabular}{|c|c|c|c|c|c|c|c|c|}
\hline TEST & $\mathrm{N}$ & MEDIA & MEDIANA & MODA & $\begin{array}{c}\text { DESV } \\
\text { EST }\end{array}$ & $\begin{array}{c}\text { PTJE } \\
\text { MÁX }\end{array}$ & $\begin{array}{c}\text { PTJE } \\
\text { MÍ́ }\end{array}$ & RANGO \\
\hline RSD (Inverso) & 88 & 8 & 8 & 7 & 2.235 & 15 & 3 & 12 \\
\hline
\end{tabular}

Respecto a la prueba Recuerdo Serial de Dígitos (Inverso), el promedio general alcanzó los 8 puntos de un total de 32, con rendimiento del 25\%. El valor central desde donde se distribuyen el resto de las puntuaciones fue 8 puntos, lo que permite determinar que el $50 \%$ de los alumnos tuvieron un puntaje igual o superior a 8. El rango entre el puntaje máximo (15) y el mínimo (3) es de 12 puntos y la disposición en las puntuaciones, respecto a la media, es en promedio $s=2,7$. Frente a estos resultados, se puede decir que esta prueba presentó un alto grado de dificultad en los niños y niñas de 8 y 9 años que cursaban Tercer Año Básico, ya que la puntuación que más se repitió fue 7 puntos, la que se encuentra bajo la media. 
Es importante destacar que este test no se realiza completo, sino que finaliza cuando el sujeto no es capaz de realizar la doble tarea de almacenamiento y procesamiento de los dígitos. De tal manera, si bien las puntuaciones se centran por sobre la media de los resultados, el promedio en sí es bajo respecto a la puntuación total de la prueba. Asimismo, por considerarse finalizado el test cuando el sujeto no logra superar una amplitud determinada, las puntuaciones de las series quedan incompletas y no con puntuación 0 . Esto explicaría porqué la moda es $7 \mathrm{si}$, el promedio del curso alcanza solo un $25 \%$ del puntaje total.

A continuación, en la Tabla 3 se presentan los valores descriptivos de la prueba Amplitud de Escuchar.

Tabla 3. Medidas de tendencia central y variabilidad del test Amplitud de Escuchar

\begin{tabular}{|c|c|c|c|c|c|c|c|}
\hline TEST & N & MEDIA & MEDIANA & MODA & $\begin{array}{c}\text { DESV } \\
\text { EST }\end{array}$ & $\begin{array}{c}\text { PUNT } \\
\text { MÁx }\end{array}$ & $\begin{array}{c}\text { PUNT } \\
\text { MÍN }\end{array}$ \\
\hline Amplitud de Escuchar & 88 & 3 & 3 & 0 & 2.23 & 9 & 0 \\
\hline
\end{tabular}

Como puede verse en la Tabla 10, los niños obtuvieron en la prueba Amplitud de Escuchar un promedio general de 3 puntos de un total de 20, con rendimiento del 15\%. El valor desde donde se distribuye el resto de las puntuaciones se centró en los 3 puntos, lo que permite determinar que el 50\% de los alumnos tuvieron un puntaje igual o superior a esta cifra. El rango entre el puntaje máximo (9) y el mínimo (0) es de 9 puntos y la disposición de las puntuaciones, respecto a la media, es en promedio $s=2.23$. Frente a estos resultados, se puede decir que esta prueba al igual que la anterior presentó un alto grado de dificultad en los niños y niñas de 8 y 9 años que cursaban Tercer Año Básico, ya que la puntuación que más se repitió fue 0 puntos, la que se encuentra 3 puntos bajo la media.

Como en la prueba Recuerdo Serial de Dígitos (Inverso), el test Amplitud de Escuchar finaliza cuando el sujeto no es capaz de realizar la doble tarea de almacenamiento y procesamiento, en este caso, de frases. De tal manera, las puntuaciones de las series que no se llegaron a realizar quedan sin puntuación y no con puntaje 0 . De igual forma, en este test, el valor que más se repitió en los resultados fue 0 , lo que indicaría que hubo mayor dificultad para contestarlo.

\section{Memoria Operativa y conciencia metapragmática: análisis correlacional}

Se han correlacionado las dos pruebas que miden memoria operativa, es decir, test Recuerdo Serial de Dígitos (inverso) y Amplitud de Escuchar obteniendo como coeficiente de correlación puntual-biserial 0.371. Esto significa que existe una asociación lineal positiva entre ambos puntajes. Al realizar la prueba de hipótesis, se obtuvo que la relación entre los puntajes del Test Recuerdo Serial de Dígitos (Inversos) y el Test Amplitud de Escuchar es estadísticamente significativa a un nivel del 1\% (Montenegro \& Pérez, 2008). 
Por otra parte, el coeficiente de correlación puntual-biserial obtenido entre los resultados de conciencia metapragmática y Recuerdo serial de dígitos (inverso) fue de 0.301 , con lo cual se concluye que existe una asociación lineal positiva entre los puntajes. Es decir, en la medida que aumenta el nivel de conciencia metapragmática aumenta también el nivel de memoria operativa. Al realizar la prueba de hipótesis (Montenegro y Pérez, 2008), se obtuvo que la relación entre los puntajes de conciencia metapragmática y la memoria operativa de de esta prueba es estadísticamente significativa a un nivel del 1\%

Finalmente, el coeficiente de correlación puntual-biserial obtenidoentre la Conciencia Metapragmática y la amplitud de Escuchar fue de 0.244 , lo que indica una asociación lineal positiva. Al realizar la prueba de hipótesis, se obtuvo que la relación entre los puntajes del instrumento CMP y el test Amplitud de Escuchar es estadísticamente significativa a un nivel del 5\%.

De acuerdo a los resultados presentados anteriormente, se puede determinar que, tanto las pruebas que miden memoria operativa (Amplitud de Escuchar y Recuerdo Serial de Dígitos Inverso) mantienen una correlación significativa entre ellas, como así también entre estos test y el instrumento CMP, que mide conciencia metapragmática. A la luz de estos resultados, en el siguiente apartado se presentan las conclusiones de esta investigación.

\section{Conclusiones}

Con este trabajo, se ha podido comprobar empíricamente que la conciencia metapragmática está relacionada con la memoria operativa, tal como lo señaló Gombert (1992). Es posible afirmar esto a partir de la correlación positiva, lineal y significativa encontrada entre las dos variables. De esta manera, el incremento de la conciencia metapragmática no dependería solamente del desarrollo lingüístico de los individuos, sino también de un crecimiento de los sistemas cognitivos involucrados en el almacenamiento y procesamiento de la información.

Al respecto, es necesario establecer que la correlación se calculó considerando todos los puntajes generales sin discriminarlos por su rendimiento. Hubiera sido interesante y, tal vez sea posible en estudios estadísticos con más población, observar qué puntajes obtienen en una determinada prueba (CMP, por ejemplo) los sujetos que tienen los puntajes menores y mayores de la otra (Memoria Operativa.).

Por otra parte, llama la atención que los resultados obtenidos por los niños en las pruebas que medían conciencia metapragmática y memoria operativa, fueran tan bajos. Daría la impresión de que se ha registrado los momentos iniciales del desenvolvimiento de estas habilidades que, posiblemente, se desarrollarán en niños con mayor edad. En lo que respecta a memoria operativa, estos resultados estarían de acuerdo con lo propuesto por Papazian, Alfonso y Luzondo (2006) 
y Baddeley (1986) en el sentido de que esta capacidad comienza a manifestarse entre los 7 y 8 años de edad, alcanzando su máximo desarrollo alrededor de los 11 años. Respecto de la conciencia metapragmática, Crespo (2008) sostiene que los niños de 8 años todavía apoyan muchas de sus respuestas en un ajuste automático epipragmático. Incluso en aquellos casos en los cuales son observadores y -por ende- se encuentran en mejores condiciones de ver dicho desajuste, ellos logran señalar el problema, pero no su origen.

Frente a este punto, es necesario considerar el desarrollo incipiente de estas habilidades con la etapa de escolaridad en la que se encuentran nuestros sujetos. Todos ellos, niños entre los 8 y 9 años de edad, han terminado la adquisición inicial de la lectoescritura y se encuentran ahora en un proceso de desarrollo ulterior de estas habilidades. A este respecto, Papazian et al. (2006), entre otros, señalan que la memoria operativa es fundamental tanto para el aprendizaje del lenguaje como para el desenvolvimiento de otros procesos cognitivos. Asimismo, Rondal, Esperet, Gombert, Thibaut y Comblain (2005) indican que la conciencia metapragmática se encuentra relacionada con el desarrollo de la lectura y la escritura. En este sentido, ambas habilidades incipientes (conciencia metapragmática y memoria operativa) son concomitantes con una alfabetización también inicial, lo que llevaría a plantearnos el rol que cumple la escuela en la emergencia y posterior desarrollo de estas habilidades o la incidencia de estas habilidades en el aprendizaje escolar.

A la luz de estas reflexiones, solo queda señalar como Crespo (2008), lo interesente que sería observar qué ocurre con estas pruebas y sus relaciones, si se aplicasen a estudiantes que están en cursos superiores de Educación Básica y Media, instancias en las cuales, por una parte, el proceso de escolarización sería más influyente y, por otra, supuestamente, los sujetos dominarían la lengua escrita de manera más acabada. Esta interrogante deberá ser resuelta por posteriores investigaciones.

\section{Bibliografía}

Acuña, X. y Sentis, F. (2004). "Desarrollo pragmático en el habla infantil". Onomazein, 10(2), 33-56.

Alsina, Á. (2007). "¿Por qué algunos niños tienen dificultades para calcular? Una aproximación desde el estudio de la memoria humana". Revista Latinoamericana de Investigación en Matemáticas Educativa, 10(3), 315-333.

Baddeley, A. \& Hitch, G. (1974). "Working Memory". En G.A Bower (Ed.), The psychology of learning and motivation (pp. 47-90). Nueva York: Academic Press.

Baddeley, A. (1986). Working Memory. Oxford: Oxford University Press. 
Baddeley, A. (1999). Memoria Humana: Teoría y Práctica. Madrid: McGrawHill.

Baqués, J. (1996). "Working memory”. En D. Sáiz, M. Sáiz y J. Baqués (Eds.), Psicología de la memoria. Manual de prácticas (pp. 14-156). Barcelona: Avesta. 14-156.

Brown, H. (1987). Principles of language and teaching. Englewood Cliffs, NJ: Prentice-Hall.

Case, R. (1985). Intellectual Development. Birth to Adulthood. New York: Academic Press.

Crespo, N. (2008). "La medición de la conciencia metapragmática de los niños: Resolviendo la ambigüedad en la comprensión oral". Revista RLA (en proceso de revisión editorial).

Crespo, N., Pérez, D. y Alfaro, P. (2008). Instrumento de evaluación de la conciencia metapragmática. CMP. Instrumento Multimedia. Manual de Aplicación Valparaíso: Litografía Garín. S.A.

Daneman, M. \& Carpenter, P. (1980). "Individual differences in working memory and reading". Journal of verbal learning and verbal Behaviour, $19,450-466$.

Engle, R., Cantor, J. \& Carullo, J.J. (1992). "Individual differences in working memory and comprehension: A test of four hypotheses". Journal of Experimental Psychology: Learning, Memory and Cognition, 18, 972-992.

Gathercole, S. \& Adams, A.M. (1994). "Children`s phonological working memory: contributions of long-term knowledge and rehearsal". Journal of Memory and Language, 32, 676-688.

Gathercole, S. \& Pickering, S. (2000). "Assessment of memory in six-and -seven- year old children”. Journal of Educational Psychology, 92(2), 377-390.

Gombert, J. (1992). Metalinguistic development. Chicago, Ill: University of Chicago Press.

Levorato, M. \& Cacciari, C. (1992). "Children's comprehension and production of idioms: the role of context and familiarity". Journal of Child Language, 19, 415-433.

Montenegro, C. y Pérez, L. (2007). Análisis Estadístico II: Test Conciencia Metapragmática. Informe interno no publicado en el marco del Proyecto FONDECYT 1070333.

Montenegro, C. y Pérez, L. (2008). Relación entre el Test de Conciencia Metapragmática y Memoria Operativa. Informe interno no publicado en el marco del Proyecto FONDECYT 1070333. 
Papazian, O., Alfonso, I. y Luzondo, R. (2006). "Trastornos de las funciones ejecutivas". Revista de Neurología, 43(3), 45-50.

Pickering, S., Baqués, J. \& Gathercole, S. (1999). Batería de Tests de Memoria de Trabajo. Barcelona: Laboratori de Memòria de la Universitat Autònoma de Barcelona (versión castellana de S. Pickering y S. Gathercole, Working Memory Battery (1997)). No comercializada.

Portolés, José (2004). Pragmática para hispanistas. Madrid: Síntesis

Ravid, Dorit \& Tolchinsky, Liliana (2002). "Developing linguistic literacy: a comprehensive model". Journal child language, 29, 417-447.

Rondal, J., Esperet, E., Gombert, J., Thibaut, J. y Comblain, A. (2005). "Desarrollo del Lenguaje Oral". En Miguel Puyuelo \& Jean A. Rondal (Edits.). Manual de desarrollo y alteraciones del lenguaje. Aspectos evolutivos y patología en el niño y el adulto (pp. 1-85). Barcelona: Masson S.A.

Salthouse, T. (1990). "Working memory as a processing resource in cognitive aging". Developmental Review, 10, 101-124.

Shaffer, D. (2000). Psicología del desarrollo. Infancia y adolescencia. México: Thompson.

Verschueren, J. (2000). "Notes on the role of metapragmatic awareness". Language use, Pragamtics, 10(4), 439-456.

Verschueren, J. (2002). Para entender la Pragmática. Madrid: Gredos. 